\title{
PERSPECTIVAS
}

Artigo convidado

DOI: http://dx.doi.org/10.159o/So034-759020170608

\section{PESQUISA EM ADMINISTRAÇÃO: EM BUSCA DE IMPACTO SOCIAL E OUTROS IMPACTOS}

\author{
Eu não estou interessado \\ em nenhuma teoria \\ em nenhuma fantasia \\ nem no algo mais... \\ Amar e mudar as coisas \\ me interessa mais... \\ Belchior
}

\section{INTRODUÇÃO}

No Encontro da Associação Nacional de Pós-Graduação e Pesquisa em Administração (ANPAD) de 2015, tive o prazer de participar de um painel organizado pelo meu colega Charles Kirschbaum, centrado no tema "Como aumentar o impacto da pesquisa em Administração". Em um auditório lotado, Charles iniciou os debates com um teste de múltipla escolha, mais ou menos nesta linha:

Se um jornalista quisesse saber algo sobre a desigualdade de salários nas organizações, para quem ligaria?

a. um economista

b. um cientista político

c. um sociólogo

d. um pesquisador em administração

A plateia, na sua maioria composta por pesquisadores em Administração, acabou maciçamente escolhendo a alternativa A. Para os presentes, o jornalista, muito provavelmente, ligaria para um economista. Certamente, a explicação para isso não é porque só os economistas fazem pesquisa sobre salários e sua distribuição nas organizações. Os determinantes de mecanismos de compensação em empresas sempre foram um tema muito comum no campo da Administração, há um bom tempo. Por exemplo, Finkelstein e Hambrick (1989) identificaram processos políticos influenciando a compensação de CEOs; Zenger (1992) usou teorias de comparação social para explicar a distribuição de salários extremos em hierarquias, no topo e na base; e Hoskisson, Castleton e Withers (2009) argumentaram que os crescentes salários recebidos por executivos de topo são

\section{SÉRGIO LAZZARINI}

sergiogl1@insper.edu.br Professor do Insper, Cátedra Chafi Haddad de Administração - São Paulo-SP, Brasil 
decorrentes de uma maior pressão de acionistas e do crescente risco que esses executivos têm que assumir nas suas empresas.

Não faltam teorias e estudos em Administração que poderiam ajudar um pesquisador a dar uma longa e inspirada entrevista sobre desigualdade de salários. Por que, então, a própria comunidade de Administração considera que não seria a primeira escolha do jornalista? Neste artigo, sem ter a pretensão de dar respostas definitivas, discutirei por que a pesquisa em Administração acaba tendo, muitas vezes, um impacto percebido aquém do seu potencial e apresentarei algumas sugestões sobre o que pode ser feito a esse respeito. Muito embora exista grande espaço para aumentar o impacto social da pesquisa na área, a partir de estudos que ajudem a entender e a solucionar questões sociais prementes, a minha discussão será um pouco mais geral: Como aumentar o impacto da pesquisa para gestores, formuladores de políticas e demais atores de entorno que possam se beneficiar dos achados trazidos por estudos teóricos ou empíricos sobre processos e estratégias organizacionais?

\section{QUAL IMPACTO?}

O impacto acadêmico de uma publicação é usualmente avaliado pelas citações que um determinado artigo ou livro recebe ao longo dos anos. Em geral, uma pesquisa academicamente relevante é aquela que ajuda a construir conhecimento em torno de um determinado tema, abordagem teórica ou objeto empírico. A geração de conhecimento, no entanto, é, por si só, um processo de construção social (Latour, 1987); é possível que determinados grupos de pesquisadores se referenciem ao longo do tempo como forma de legitimar ou consolidar um determinado campo, ainda que esteja divorciado da realidade ou de qualquer tipo de aplicação. Uma pergunta-chave que emerge nesse contexto é: Será que uma pesquisa prestigiada por pares acadêmicos também resulta em maior relevância para compreender ou solucionar questões reais?

Para examinar essa questão, Baldridge, Floyd e Markóczy (2004) obtiveram uma amostra aleatória de 120 artigos publicados em revistas acadêmicas de alto impacto em Administração. Em linha com a discussão anterior, o impacto acadêmico dos artigos foi medido pelo seu volume de citações futuras. Para avaliar o impacto dos artigos no mundo empresarial, os autores construíram um índice de "relevância" avaliado por um painel de experts com atuação executiva em organizações. O resultado: os autores encontraram uma correlação estatisticamente significante entre a relevância do artigo e o seu impacto acadêmico. Aparentemente, os artigos mais citados foram, em geral, considerados pelos executivos como de maior potencial para gerar lições relevantes para o mundo empresarial. Uma possível explicação é que artigos mais citados tendem a ser aqueles de maior qualidade e com resultados mais fortes e surpreendentes, com amplo potencial de contestar visões preestabelecidas ou lançar luz sobre novos conceitos ou relações entre variáveis de alta relevância.

É claro que esses resultados se baseiam em correlações. Os autores citam o caso de artigos julgados relevantes pelos executivos, mas que não receberam uma única citação. Há, também, artigos muito citados e julgados pouco relevantes esses podem ser aqueles ligados a debates teóricos cerceados no mundo dos pesquisadores e com pouca ancoragem a problemas reais. Por certo, há debates teóricos que não trazem aplicação imediata, porém abrem espaço para avanços aplicados relevantes. Por exemplo, a discussão teórica trazida por Granovetter (1985), apresentando sua perspectiva de embededdness em oposição a abordagens com raiz em racionalidade econômica, foi fundamental para abrir toda uma nova linha de pesquisa aplicada sobre a governança social de alianças e redes de empresas. A chave, aqui, está em construir debates de alto impacto acadêmico, mas que efetivamente gerem avanços no conhecimento relevante.

Nesse sentido, McGahan (2007) traz uma série de exemplos e sugestões sobre como aumentar a relevância gerencial da pesquisa em Administração. Uma possibilidade é buscar resultados surpreendentes que sejam, a princípio, contraintuitivos, mas que possam abrir novas oportunidades de refinamento teórico. Por exemplo, Khanna e Palepu (2000) buscaram mostrar que a visão tradicional sobre os malefícios de diversificação excessiva não é necessariamente verdadeira em países carentes de boas instituições e mercados eficientes. Esse ponto acabou gerando toda uma nova literatura sobre estratégia em países emergentes e rapidamente passou a ser usado como justificativa gerencial para a montagem (ou a permanência) de grupos empresariais altamente diversificados, a despeito do que ensina a maior parte dos livros-texto de estratégia.

\section{IMPACTO SOCIAL E NO DEBATE PÚBLICO}

Há, adicionalmente, uma crescente preocupação de gerar pesquisas que ajudem a entender problemas sociais complexos e proponham possíveis encaminhamentos, senão soluções, para esses problemas. Uma questão muito comumente examinada, por exemplo, é como empresas podem atender objetivos sociais e ambientais para além do exigido pelos seus acionistas. Desenvolveu-se, em Administração, um volumoso corpo de estudos teóricos e empíricos tentando examinar o papel de 
diversos stakeholders na concepção das estratégias das empresas e no seu desempenho final (Barnett, 2007; Freeman, 1984; Garcia-Castro \& Aguilera, 2015). Há, também, pesquisas mais diretamente ligadas ao estudo de organizações com objetivos eminentemente de impacto social ou ambiental, que podem ou não buscar lucratividade ou sustentabilidade financeira como objetivo conjunto (Battilana \& Dorado, 2010; Comini, Barki, \& Aguiar, 2012). Mais ainda, pesquisadores têm examinado as complexas interações entre atores públicos e estatais na concepção e execução de projetos de interesse público (Cabral, Lazzarini, \& Azevedo, 2013; Kivleniece \& Quelin, 2012; Mahoney, McGahan, \& Pitelis, 2009).

A despeito da importância dessas linhas de investigação, uma pesquisa de alto impacto em Administração não precisa necessariamente ser direcionada ao estudo de organizações socialmente engajadas ou com interface pública. Seguindo a minha discussão anterior, uma pergunta mais básica é se a pesquisa está efetivamente contribuindo para gerar resultados relevantes para o mundo gerencial. É curioso, por exemplo, observar acadêmicos criticando determinados modelos de amplo uso por gestores, mas que, na sua atividade de ensino, acabam reforçando essas próprias teorias que tanto criticam! Alguns professores de estratégia, por exemplo, denunciam as limitações da abordagem estrutural de Porter (1980), porém usam justamente o seu famoso "modelo cinco forças" para ensinar, a alunos de graduação e MBA, formas de analisar indústrias e setores. Se essa abordagem é falha, qual alternativa poderia ser usada para capacitar e auxiliar profissionais de gestão?

Além de deixar mais claro como as pesquisas podem informar ou até mesmo mudar práticas de gestão, há, também, grande espaço para tornar o campo acadêmico de Administração mais próximo do debate público. 0 caso que reportei na introdução demonstra que há, aparentemente, uma lacuna entre o que é feito em pesquisa em Administração e o que efetivamente é utilizado ou considerado em discussões mais abertas com a sociedade. Porém, aqui não parece haver um problema de repertório; há uma grande diversidade de estudos em Administração tangenciando inúmeros temas que usualmente aparecem no debate público. Parece haver simplesmente falta de diálogo, de um maior esforço para conectar os resultados das pesquisas e as discussões aplicadas sobre temas relevantes e atuais.

Considere, por exemplo, um tópico discutido à exaustão quando se fala sobre as causas do baixo crescimento da economia brasileira: produtividade. Em tese, esse seria um tema sobre o qual os pesquisadores em Administração deveriam ter muito a dizer, especialmente porque, cada vez mais, se fala em como aumentar a produtividade da economia por meio de melhores práticas de gestão nos setores público ou privado. Curiosamente, nesse campo, os economistas parecem ter tomado muito mais espaço no debate que os acadêmicos de Administração, ainda que esses últimos continuem criticando os primeiros por uma visão excessivamente limitada e reducionista das organizações. Os trabalhos de Bloom e Van Reenen (2010), por exemplo, mostraram como práticas gerenciais se correlacionam com maior produtividade das empresas em diversos países. Recentemente, os resultados desses estudos foram tomados por acadêmicos de estratégia propondo uma "visão baseada em práticas" (Bromiley \& Rau, 2014).

Por que, com raras exceções, os acadêmicos em Administração não participam mais do debate sobre produtividade? Em algumas conversas informais, já notei uma certa aversão conceitual ao tema, que denotaria, para alguns, uma visão antiga (taylorista) de gestão. Há, aqui, uma falha de entendimento. Produtividade é apenas uma medida de desempenho (o quanto determinados recursos geram de produto ou riqueza, tudo o mais constante). Maior desempenho, por sua vez, pode ser gerado por um conjunto de práticas distintas, desde as tayoloristas clássicas (padronização de processos e monitoramento) até aquelas tidas como mais modernas (decisão em times, processos de construção coletiva de conhecimento, gestão de redes organizacionais e assim por diante). Ao virar as costas para o tema da produtividade, os acadêmicos de Administração perdem a oportunidade de fazer contribuições fundamentais ao conhecimento e à prática. Será que as medidas de qualidade de gestão empregadas pelos economistas são adequadas? Mesmo que essas práticas existam e aumentem a produtividade, quais seriam os processos políticos, sociais ou cognitivos que impediriam a sua adoção? Essas são perguntas muito afeitas ao métier da pesquisa em Administração, mas que raramente aparecem no debate público sobre como aumentar a produtividade das empresas.

\section{PUBLICAÇÃO INTERNACIONAL É INCONSISTENTE COM IMPACTO LOCAL?}

Um debate polêmico ligado aos dilemas de impacto da pesquisa em Administração diz respeito ao direcionamento das publicações. Respondendo a críticas de que poucos pesquisadores em Administração buscam publicar em revistas de elevado impacto no exterior, alguns acadêmicos argumentam que pesquisas de maior relevância focalizam aspectos locais, o que acaba reduzindo a sua aceitação em periódicos estrangeiros. Um corolário dessa afirmação é que a disseminação de pesquisa 
em contextos internacionais desvaloriza revistas construídas e geridas pela comunidade local. Essas revistas, em tese, teriam mais impacto no debate nacional do que veículos direcionados a acadêmicos de outros países.

Há várias ressalvas a esse tipo de argumento. Primeiro, será que a pesquisa que é publicada em periódicos locais é efetivamente usada para a construção de conhecimento por acadêmicos do país? O que se vê, em vários casos, são pesquisas publicadas localmente, mas que buscam simplesmente aplicar ou testar teorias internacionalmente desenvolvidas. Para que as pesquisas locais se insiram nas redes globais de geração de conhecimento, é importante que uma parte delas tente mirar periódicos acessados e lidos por uma comunidade mais ampla. Sabe-se, por exemplo, que a probabilidade de um artigo ser usado e citado por outros acadêmicos aumenta quando é publicado em periódicos de alto prestígio (Judge, Cable, Colbert, \& Rynes, 2007). Os pesquisadores em geral simplesmente não conseguem acompanhar as dezenas de revistas locais ou internacionais na sua área; sua atenção, portanto, naturalmente recai sobre os periódicos mais importantes. Mais ainda, a própria publicação da pesquisa em um veículo de destaque já serve como um sinal (ainda que imperfeito) da sua qualidade, inovação e rigor. Se os resultados de Baldridge et al. (2004), citados antes, se mantiverem corretos, esse atributo também deverá se correlacionar com a relevância percebida da pesquisa.

Segundo, não conheço evidência substantiva de que a maior parte das publicações em periódicos locais seja efetivamente utilizada por gestores ou ajude a iluminar questões prementes no debate aplicado (Cabral \& Lazzarini, 2011). Se um artigo local simplesmente toma alguma abordagem teórica já estabelecida e a ilustra com casos ou dados nacionais, nada garantirá achados ou conclusões surpreendentes que chamem a atenção de um público mais amplo. Além disso, não há nada que impeça que uma pesquisa publicada internacionalmente seja amplamente divulgada no contexto local e sirva como insumo para importantes discussões gerenciais ou de políticas públicas.

Dito isso, é inegável que existem temas locais cuja relevância pode ser pouco compreendida por editores e revisores de artigos submetidos a publicação internacional. Nesse sentido, as revistas nacionais e outros veículos (como livros de maior divulgação publicados no país) sempre continuarão sendo muito importantes. Mas isso não implica que achados locais não possam receber destaque no circuito global. A saída, nesse caso, é tentar encontrar problemas de elevada relevância local cuja análise rigorosa consiga trazer resultados surpreendentes e inovadores, resultados esses que, quem sabe, possam até mesmo desafiar conceitos e teorias tradicionalmente aceitas (Lazzarini, 2012). O exemplo anterior das pesquisas identificando as implicações estratégicas para empresas localizadas em países com instituições mais fracas ilustra bem esse ponto. Com o avanço dos países emergentes e os seus enormes desafios, até mesmo acadêmicos nos eixos de pesquisa tradicionais passaram a se interessar por fenômenos como a prevalência de grupos diversificados (Wan \& Hoskisson, 2003), a presença do Estado como acionista (Cuervo-Cazurra, Inkpen, Musacchio, \& Ramaswamy, 2014), os efeitos da corrupção (Jeong \& Weiner, 2012), modelos de negócios desenhados para atender consumidores na base da pirâmide (Prahalad, 2004), entre vários outros.

\section{EM BUSCA DE MAIS IMPACTO}

São vários os possíveis caminhos para aumentar o impacto da pesquisa em Administração. Sem a pretensão de ser exaustivo, encerro 0 artigo com algumas sugestões, muitas delas resultantes da minha própria experiência. Um ponto importante é sempre tentar ancorar desenvolvimentos teóricos e testes empíricos a um problema central, de elevada relevância no mundo gerencial ou no campo das políticas públicas. Naturalmente, nós nos apegamos a determinadas vertentes teóricas, com os seus pressupostos e implicações. Nós nos autodenominamos especialistas em teoria institucional, abordagem dos stakeholders ou visão baseada em recursos. Mas essas teorias não existem em um vácuo; elas, em geral, resultam da necessidade de entender melhor determinados fenômenos. Por exemplo, no desenvolvimento da sua teoria dos custos de transação, Williamson (1985) sempre deixou claro que seu problema focal era explicar padrões de integração vertical das firmas - um tema polêmico, dado que a presença das empresas em múltiplos elos da cadeia produtiva poderia resultar em efeitos anticompetitivos. Williamson buscou enfatizar possíveis ganhos de eficiência trazidos pela integração vertical das firmas, em função de diversos fatores e contingências (como o grau de especificidade dos ativos). Apesar de ter foco econômico, a pesquisa de Williamson teve alta repercussão em comunidades de gestão interessadas em explicar e entender estratégias de expansão corporativa nas cadeias produtivas.

Também pode ser muito importante manter contato próximo com empresas, institutos e governos, trazendo questões e problemas que possam representar oportunidades concretas de pesquisa. Mas há, aqui, diversos cuidados a serem tomados. Muitas organizações demandam consultoria para soluções que resultam de aplicação direta de modelos ou metodologias já conhecidas e estabelecidas, para as quais pouco esforço de 
pesquisa é necessário. Porém, a academia realmente adicionará valor quando auxiliar essas organizações a compreender ou solucionar questões novas e cujo esforço analítico permitirá melhor compreender determinados fenômenos aplicados. Em se firmando parcerias com empresas e governos, é importante também garantir que os pesquisadores tenham autorização para publicar os resultados e achados dos projetos, e que eventuais financiamentos externos desses projetos não comprometam a imparcialidade e isenção dos pesquisadores.

Além disso, um exame atento a atividades e setores ainda pouco estudados pode trazer oportunidades concretas de inovar, com novas perspectivas e abordagens. Em 2010, Jay Barney e Anita McGahan organizaram um workshop na Ohio State University para discutir como pesquisadores de Administração poderiam contribuir para debates de políticas públicas. Estava presente no evento William Ouchi, um acadêmico de grande importância no desenvolvimento de teorias organizacionais (Ouchi, 1980) e que, mais recentemente, passou a atuar com gestão educacional (Ouchi, 2008). Ouchi disse que, se fosse um aluno de doutorado ou professor em início de carreira, construiria sua agenda de pesquisa estudando organizações atípicas, como escolas, prisões e hospitais. São esses contextos, segundo ele, que possivelmente trarão novos elementos e achados para além do que já foi estudado em setores tradicionais. Porém, até mesmo empresas estabelecidas, em indústrias tradicionais, passam, cada vez mais, a implantar ações para reduzir o impacto ambiental das suas atividades, aumentar a diversidade de gênero e racial dos seus executivos, lidar com políticas governamentais e distribuir valor para parceiros ao longo das suas cadeias produtivas. São todos temas inovadores, relevantes gerencialmente, de alto interesse para o debate público e que, de quebra, podem representar grandes oportunidades de construção e refinamento teórico.

Dessa forma, não precisamos nem devemos abandonar ou desmerecer teorias, como dito na epígrafe deste artigo. Mas, certamente, com um novo esforço investigativo, podemos direcioná-las para temas prementes e pouco estudados. E, quem sabe, mudar as coisas para melhor.

\section{REFERÊNCIAS}

Baldridge, D. C., Floyd, S. W., \& Markóczy, L. (2004). Are managers from Mars and academicians from Venus? Toward an understanding of the relationship between academic quality and practical relevance. Strategic Management Journal, 25(11), 1063-1074. doi:10.1002/smj.406

Barnett, M. L. (2007). Stakeholder influence capacity and the variability of financial returns to corporate social responsibility. Academy of Management Review, 32(3), 794-816.
Battilana, J., \& Dorado, S. (2010). Building sustainable hybrid organizations: The case of commercial microfinance organizations. Academy of Management Journal, 53(6), 1419-1440. doi:10.5465/ amj.2010.57318391

Bloom, N., \& Van Reenen, J. Van. (2010). Why do management practices differ across firms and countries? The Journal of Economic Perspectives, 24(1), 203-224.

Bromiley, P., \& Rau, D. (2014). Towards a practice-based view of strategy. Strategic Management Journal, 35(8), 1249-1256. doi:10.1002/ smj. 2238

Cabral, S., \& Lazzarini, S. G. (2011). Internacionalizar é preciso, produzir por produzir não é preciso. Organizações \& Sociedade, 18(58), 541. 542. doi:10.1590/S1984-92302011000300011

Cabral, S., Lazzarini, S. G., \& Azevedo, P. F. (2013). Private entrepreneurs in public services: A longitudinal examination of outsourcing and statization of prisons. Strategic Entrepreneurship Journal, 7(1), 6-25. doi:10.1002/sej.1149

Comini, G., Barki, E., \& Aguiar, L. T. Dd. (2012). A three-pronged approach to social business: A Brazilian multi-case analysis. RAUSPRevista de Administração da Universidade de São Paulo, 47(3), 385397. doi:10.1590/Soo80-21072012000300004

Cuervo-Cazurra, A., Inkpen, A., Musacchio, A., \& Ramaswamy, K. (2014). Governments as owners: State-owned multinational companies. Journal of International Business Studies, 45(8), 919-942. doi:10.1057/jibs.2014.43

Finkelstein, S., \& Hambrick, D. C. (1989). Chief executive compensation: A study of the intersection of markets and political processes. Strategic Management Journal, 10(2), 121-134. doi:10.1002/ smj.4250100203

Freeman, R. E. (1984). Strategic management: A stakeholder approach. London, UK: Pitman.

Garcia-Castro, R., \& Aguilera, R. V. (2015). Incremental value creation and appropriation in a world with multiple stakeholders. Strategic Management Journal, 36(1), 137-147. doi:10.1002/smj.2241

Granovetter, M. (1985). Economic action and social structure: The problem of embeddedness. American Journal of Sociology, 91(3), 481-510.

Hoskisson, R. E., Castleton, M. W., \& Withers, M. C. (2009). Complementarity in monitoring and bonding: More intense monitoring leads to higher executive compensation. The Academy of Management Perspectives, 23(2), 57-74. doi:10.5465/ amp.2009.39985541

Jeong, Y., \& Weiner, R. J. (2012). Who bribes? Evidence from the United Nations' oil-for-food program. Strategic Management Journal, 33(12), 1363-1383. doi:10.1002/smj.1986

Judge, T. A., Cable, D. M., Colbert, A. E., \& Rynes, S. L. (2007). What causes a management article to be cited: Article, author, or journal? Academy of Management Journal, 50(3), 491-506. doi:10.5465/ amj.2007.25525577

Khanna, T., \& Palepu, K. (2000). The future of business groups in emerging markets: Long-run evidence from Chile. Academy of Management Journal, 43(3), 268-285.

Kivleniece, I., \& Quelin, B. V. (2012). Creating and capturing value in public-private ties: A private actor's perspective. Academy of Management Review, 37(2), 272-299. doi:10.5465/amr.2011.0004 
Latour, B. (1987). Science in action: How to follow scientists and engineers through society. Cambridge, USA: Harvard University Press.

Lazzarini, S. G. (2012). Leveraging the competitive advantage of Iberoamerican scholars. Management Research: Journal of the Iberoamerican Academy of Management, 10(1), 64-73. doi:10.1108/1536-541211228577

Mahoney, J. T., McGahan, A. M., \& Pitelis, C. N. (2009). The interdependence of private and public interests. Organization Science, 20(6), 1034-1052. doi:10.1287/orsc.1090.0472

McGahan, A. M. (2007). Academic research that matters to managers: On zebras, dogs, lemmings, hammers, and turnips. Academy of ManagementJournal,5o(4), 748-753. doi:10.5465/amj.2007.26279166

Ouchi, W. G. (1980). Markets, bureaucracies, and clans. Administrative Science Quarterly, 25(1), 129-141. doi:10.2307/2392231
Ouchi, W. G. (2008). Power to the principals: Decentralization in three large school districts. Organization Science, 17(2), 298-307. doi:10.1287/orsc.1050.0172

Porter, M. E. (1980). Competitive strategy. New York, USA: The Free Press.

Prahalad, C. K. (2004). The fortune at the bottom of the pyramid. Upper Saddle River, USA: Pearson.

Wan, W. P., \& Hoskisson, R. E. (2003). Home country environments, corporate diversification strategies, and firm performance. Academy of Management Journal, 46(1), 27-45.

Williamson, O. E. (1985). The economic institutions of capitalism. New York, USA: The Free Press.

Zenger, T. R. (1992). Why do employers only reward extreme performance? Examining the relationships among performance, pay, and turnover. Administrative Science Quarterly, 37(2), 198-219. doi:10.2307/2393221 